\title{
Assess the Effect of Intradialytic Stretching Exercises to Reduce Leg Muscle Cramps among Patients Undergoing Hemodialysis in Selected Dialysis Unit of Ernakulum District
}

\author{
Jancy $\mathrm{PO}^{1, *}$, and Parimala $\mathrm{S}^{2}$ \\ ${ }^{1} \mathrm{PhD}(\mathrm{N})$ Scholar, Vinayaka Mission's Research foundation (Deemed to be University) Salem, Tamil Nadu, India \\ ${ }^{2}$ Research Supervisor, Vinayaka Mission's Research foundation (Deemed to be University), Salem, Tamil Nadu, India
}

*Corresponding author: Jancy PO, PhD (N) Scholar, Vinayaka Mission's Research foundation (Deemed to be University) Salem, Tamil Nadu, India, E-mail: jencytherese@yahoo.com

Received: 15 Jun, 2020 | Accepted: 02 Jul, 2020 | Published: 08 Jul, 2020

Citation: Jancy PO, Parimala S (2020) Assess the Effect of Intradialytic Stretching Exercises to Reduce Leg Muscle Cramps among Patients Undergoing Hemodialysis in Selected Dialysis Unit of Ernakulum District. Int J Nephrol Kidney Fail 6(3): dx.doi.org/10.16966/2380-5498.198

Copyright: (c) 2020 Jancy PO, et al. This is an open-access article distributed under the terms of the Creative Commons Attribution License, which permits unrestricted use, distribution, and reproduction in any medium, provided the original author and source are credited.

\section{Introduction}

Integrated functioning of each organs of our human body helps to lead a healthy life in the world. The kidney has a vital role to keep our body healthy [1]. In modern era, Chronic Kidney Disease (CKD) is one of the serious public health problems among all life style diseases $[2,3]$. CKD is accompanying with end-stage renal disease (ESRD) and escalates morbidity, mortality and cost of the health care system in the developing countries [4-6].

Early detection and implementation of therapeutic approaches have been accentuated to delay the advancement of disease and improve the quality of life [7].

Advancement of CKD leads to renal failure which requires expensive renal replacement therapies [8]. Hemodialysis is being the most common and effective treatment for renal failure [9]. 9,20,000 people are undergoing hemodialysis per day in the world, i.e., $7.8 \%$ of the total population [10]. Globally $10 \%$ of the population is suffering from CKD and millions are dying each year due to the lack of costeffective treatment [11].

Global ranking of premature causes of death (2010) reveals that the kidney disease rising from 32 in 1990 to 24 in 2010. 55,000 dialysis patients are in India and the ratio is increasing from 10-20\% annually [12]. Global burden of chronic renal failure is approximately $1,1010,107$ and 8,50,000 death per year. In India the incidence of renal failure is $0.7 \%$ or 7852 per million per population [13]. There are about 2,500,000 of people suffering with kidney diseases in Kerala, as per the statistics of the government agency "Mrithasanjeevani" (2019). 1450 patients are waiting for kidney transplantation. There was 11.5 times increase in hemodialysis sessions from 2008 to 2017 in Kerala.

Hemodialysis is one of the most important treatment modality for CKDs. It is an ongoing process with patients experiencing various complications like hypotension, muscle cramps, disequilibrium syndrome and nausea during the procedure. Muscle cramps are mostly seen in these patients who begin with very painful muscle twitches and hard movements. Calf muscle, feet, toes, thighs and abdomen are the usual sites for muscle cramps. 25\%-50\% of dialysis patients are affected with muscle cramps during or after hemodialysis [14]. It occurs due to decreased-fluid volume, blood pressure, sodium levels, fluid and electrolyte imbalance, tissue hypoxia and hypomagnesaemia. Too much dialysis ultrafiltration and raising of serum creatinine kinase may cause an abnormal use of energy by muscle and therefore leading to muscle cramps.

Exercise is one of the probable preventive measures to decrease the loss of muscle protein and maintenance of muscle functions. In recent times, several studies have revealed the importance of exercises and physical activities to cease muscle wasting in ESRD patients. Patients recommended for intradialytic exercise, which seems to be physically active $[15,16]$. Intradialytic exercise also helps for improving their quality of life and exercise tolerance [17-19].

Physical activity programs could be divided into separate exercise according to the availability of time within the complex hemodialysis treatment. Each one of the activity programs is distinctive due to diverse acceptance of the treatment complexity and the disease itself [20].

A famous German Nephrologist divided physical activity programs into numerous groups consistent with the specific concern on individual components of physical fitness, training of balance, co-ordination, breathe exercise, relaxation techniques, isometric exercise and stretching exercise during hemodialysis. The goal of stretching exercise is to improve general muscle strength, enhance muscle volume, decrease muscle fiber's atrophy, support bone tissue, mineralization and reduce the muscle cramps. Dialysis Nurses are 
in a unique position to help monitor and evaluate cramps and in management techniques [21].

\section{Statement of the Problem}

A study to assess the effect of Intradialytic stretching exercises to reduce leg muscle cramps among patients undergoing hemodialysis in selected dialysis unit of Ernakulum district, Kerala.

\section{Objectives}

- $\quad$ Assess the severity of leg muscle cramps among patients undergoing hemodialysis

- $\quad$ Assess the effect of Intradialytic stretching exercises on leg muscle cramps among patients undergoing hemodialysis.

\section{Hypothesis:H1}

There is a significant reduction in leg muscle cramps among patient's undergone Intradialytic stretching exercises.

\section{Research approach}

Quantitative research approach was used to assess leg muscle cramps.

\section{Research design}

The research design adopted for this study was Quasi experimental. Pre-test and post-test experimental design was used.

\section{Variables}

- Independent variable: Intradialytic stretching exercises

- $\quad$ Dependent variable: Leg muscle cramps

\section{Setting of the study}

Study was conducted in the dialysis unit of Koinonia Mission Hospital, Allapra, Ernakulum district Kerala state.

\section{Study population}

Population is the aggregation of all the units in which a researcher is interested. In the present study, the population consisted of patients undergoing hemodialysis who are experiencing leg muscle cramps.

\section{Sample}

Sample means the patients undergoing hemodialysis experiencing leg muscle cramps in selected dialysis unit of Ernakulum district, Kerala.

\section{Sample size}

30 Patients undergoing hemodialysis in the selected dialysis unit of Ernakulum district.

\section{Sampling technique}

Random sampling.

\section{Sampling criteria}

\section{Inclusion criteria}

1. Patients who are undergoing hemodialysis for 4 hours.

2. Patients who are undergoing hemodialysis from 4 months to 3 years.

3. Patients who are willing to participate in the study.

4. Patients experiencing muscle cramps during and after dialysis.
Exclusion criteria

1. Patients who are unable to communicate.

2. Patients who are having breathing difficulty.

Tools

\section{Development or selection of the tool}

The investigator selected the tools on the basis of review of literature and in consultation with experts in this field.

\section{Description of tool}

The tools for the study consist of structured questionnaire to assess socio demographic data and cramps questionnaire, standardized pain scale to assess the level of pain.

\section{Tool 1: Demographic variables}

This section consist of demographic data such as age, gender, occupation, education, duration of illness, number of hemodialysis per week, hours of dialysis treatment, time and location of muscle cramps experience, deterioration of quality of life.

\section{Tool 2: Cramp questionnaire chart}

This section consists of 5 questions with different options to assess the leg muscle cramps. Total score of the cramps questionnaire is 13. Minimum score is 0 (No cramps), maximum score is 13 (Severe cramps).

The use of pain intensity scale is an easy and reliable method of determining the client's pain intensity. In this study, the 11 point pain intensity scale with word modifiers is used to assess the pain level. The results have been incorporated into the cramp questionnaire.

\section{Content validity}

Content validity is concerned with the degree to which an instrument has an appropriate sample of items for the construct being measured. To ensure content validity of the tool-Cramp questionnaire along with demographic data was submitted to 5 experts from Medical Surgical Nursing and Community Health Nursing speciality. According to their suggestions, modifications were done.

\section{Reliability of the tool}

Reliability is the degree of consistency and accuracy with which an instrument measures the attributes designed to measure. In this study, reliability was checked using test retest method. The tool was found to be reliable (0.716).

\section{Data collection process}

The investigator obtained a formal written permission from the concerned authorities to conduct the main study. The study was conducted in dialysis unit of Koinonia Mission Hospital, Allapra from $2 / 12 / 19$ to $20 / 12 / 19$. The investigator explained the purpose of the study to the samples and informed consent was obtained. Confidentiality was assured. A questionnaire on demographic data and cramps were administered for participants to obtain information regarding leg muscle cramps. Pretest was done by using cramp questionnaire chart and numerical pain scale. Stretching exercises were given and the posttest was conducted after 6 sittings of dialysis.

\section{Plan for data analysis}

Data was planned and analyzed based on objective and hypothesis of the study and organized in following manner. 


\section{Section 1}

Demographic data were analyzed in terms of frequency and percentage.

\section{Section 2}

Pre-test and post-test level of muscle cramps were analyzed by cramp questionnaire in terms of frequency and percentage.

\section{Section 3}

Effectiveness of Intradialytic stretching exercise on muscle cramps were analyzed by paired' test.

\section{Analysis and Interpretation}

Analysis and interpretation of the data is the most important phase of research process, which involves the computation of the certain measures along with searching for patterns of relationships that exist among data groups.

\section{Organization of data}

The data was collected, tabulated, analyzed, interpreted and the results obtained were organized in the following three sections.

\section{Section 1}

Description of sample characteristics in terms of percentage and frequency.

\section{Section 2}

Description of sample score on pre-test and post-test level of leg muscle cramps by means of percentage and frequency.

\section{Section 3}

Effectiveness of Intradialytic stretching exercises on reduction of leg muscle cramps are analyzed by using paired t' test.

\section{Results}

Among 30 samples, $33 \%$ belonged to the age group of $41-50$ years and $83 \%$ samples were males.

Considering the educational status, $43 \%$ were educated up to SSLC. $43 \%$ of them have been suffering from this illness for more than 2 years. Majority of the samples, i.e. $83 \%$ were coming for hemodialysis thrice in a week and $47 \%$ of them were undergoing dialysis from 4 months to 3 years. $83 \%$ of samples experienced muscle cramps after dialysis. $67 \%$ encountered activity restriction due to muscle cramps and $80 \%$ had muscle cramps over calf muscles. The quality of life was affected due to muscle cramps for $67 \%$. The pretest cramp questionnaire revealed that $57 \%$ had moderate level of muscle cramps. The Posttest cramp questionnaire results showed that $43 \%$ samples had no muscle cramps. There is a significant difference in the pretest and posttest level of leg muscle cramps.

\section{Section 1}

Line diagram showing distribution of samples based on the time of experiencing leg muscle cramps shows that $83 \%$ of sample experiences muscle cramps after dialysis (Figure 1).

Column diagram showing distribution of samples based on restricted activity due to muscle cramps depicts that $67 \%$ of sample had restricted activities due to muscle cramps (Figure 2).

Pie diagram showing the distribution of samples based on affected leg depicts that $67 \%$ of sample has muscle cramps on both legs (Figure 3).
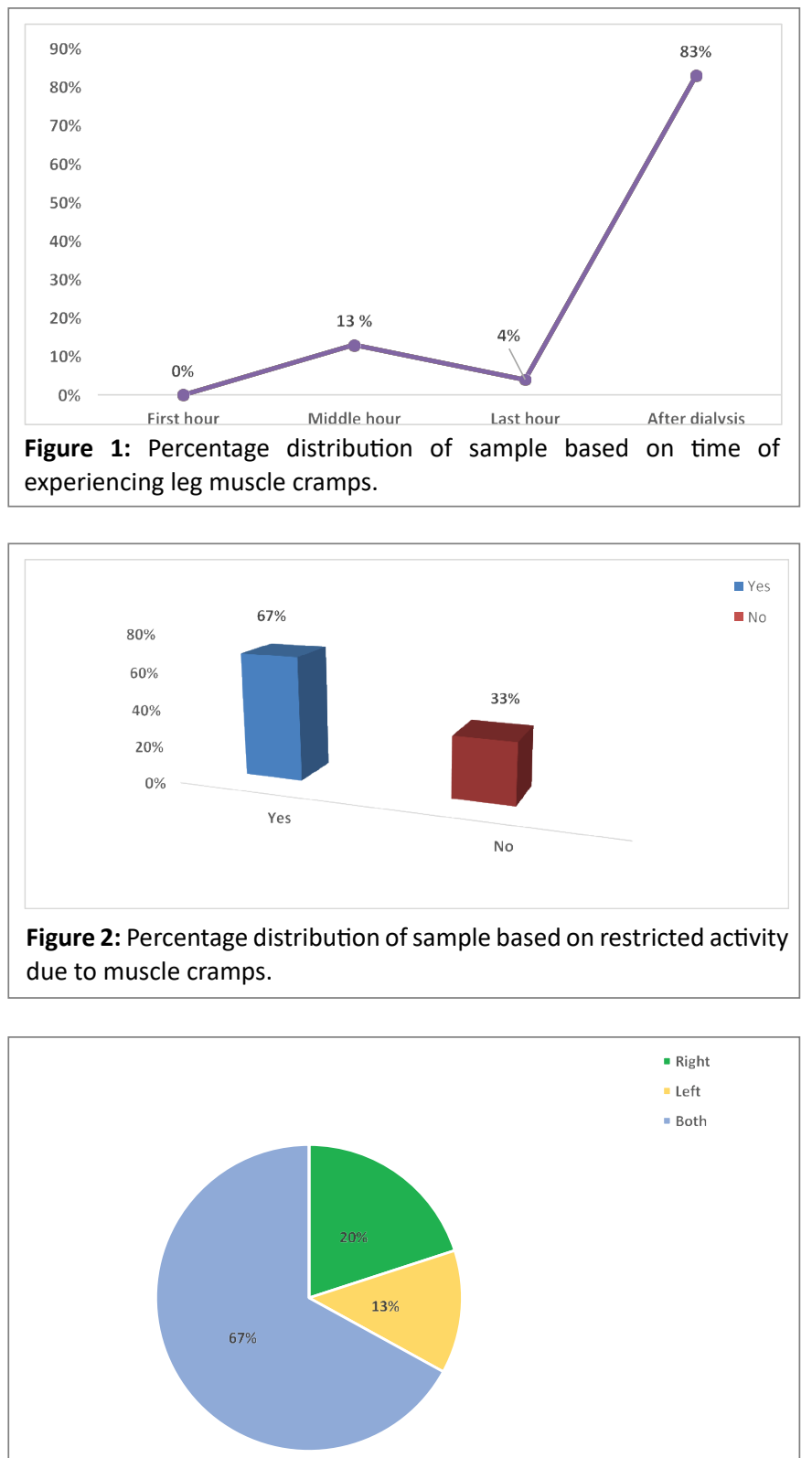

Figure 3: Percentage distribution of sample based on affecting legs.

Bar diagram showing the distribution of sample based on muscle involvement reveals that most $(80 \%)$ of them experienced muscle cramps on calf muscle (Figure 4).

Doughnut diagram showing the distribution of sample based on deterioration in quality of life reveals that majority (67\%) of them had deterioration in the quality of life due to muscle cramps (Figure 5).

\section{Section 2}

This section deals with the cramp questionnaire score of sample.

Table 1 show that in pretest $43 \%$ of samples had severe muscle cramps and $57 \%$ had moderate level of muscle cramps. Where as in posttest only $3 \%$ had severe cramps and majority that is $43 \%$ had no complaints of muscle cramps, which shows that the Intradialytic stretching exercises are effective. 


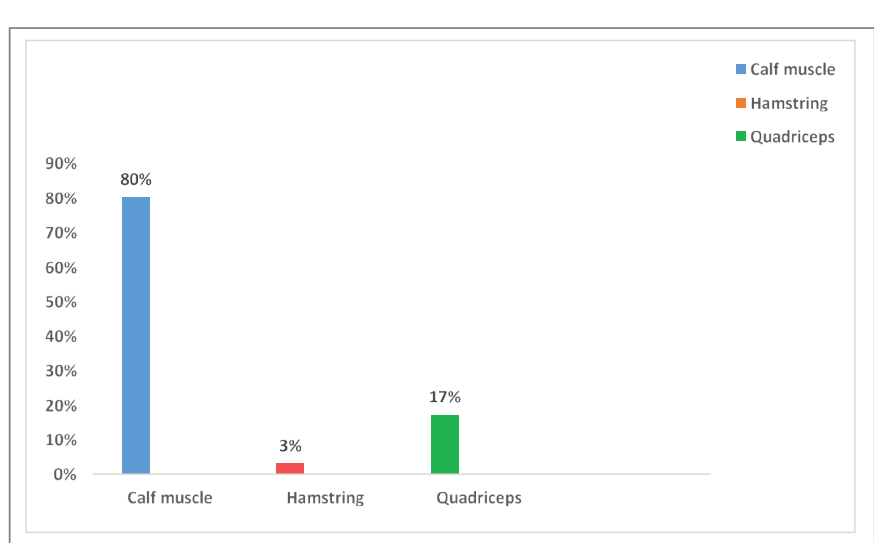

Figure 4: Percentage distribution of sample based on muscle involvement.

\begin{tabular}{|l|}
\hline \\
Figure 5: Percentage distribution of sample based on deterioration in \\
the quality of life.
\end{tabular}

\section{Section 3}

This section deals with effectiveness of Intradialytic stretching exercises on patients with leg muscle cramps.

The table 2 reveals that the mean score of leg muscle cramps of patients undergoing hemodialysis is reduced in posttest $(3.1 \pm 3.6)$ than in pretest $(8.3 \pm 3.6)$. The calculated paired t-value is 7.73 and table value is 2.05 at 0.05 level of significance. Hence the hypothesis (H1) is accepted. It is inferred that Intradialytic stretching exercises are effective to reduce leg muscle cramps in patients undergoing hemodialysis.

\section{Discussion}

The present study aimed at assessing the effect of Intradialytic stretching exercises on reduction of leg muscle cramps among patients undergoing hemodialysis. Pretest level of muscle cramps was 8.3 and the posttest value was 3.1. The finding reveals this study was effective and paired $t$ test value for 29 degree of freedom (n-1) is 7.73 which is greater than table value and is found to be significant at the level of 0.05 .

These findings were in line with the results of a study performed by Mohamed M, et al. [22] who found that performing Intradialytic stretching exercises reduces the level of muscle cramps and were statistically significant with the level of significance at $p<0.059$. More than half of the patients experienced muscle cramps in calf muscles. The study findings also revealed that there was statistically significant difference in reducing the frequency of muscle cramps at $p<0.05$ level
Table 1: Distribution of sample based on pretest and posttest level of leg muscle cramps based on cramp questionnaire score.

\begin{tabular}{|l|l|c|c|c|c|}
\hline S.No & $\begin{array}{c}\text { Level of } \\
\text { muscle cramps }\end{array}$ & Pre test & & Post test & \\
\hline & & Frequency & Percentage & Frequency & Percentage \\
\hline 1 & No cramps & 0 & $0 \%$ & 13 & $43 \%$ \\
\hline 2 & Mild cramps & 0 & $0 \%$ & 6 & $20 \%$ \\
\hline 3 & $\begin{array}{l}\text { Moderate } \\
\text { cramps }\end{array}$ & 17 & $57 \%$ & 10 & $33 \%$ \\
\hline 4 & Severe cramps & 13 & $43 \%$ & 1 & $3 \%$ \\
\hline
\end{tabular}

Table 2: Comparison of pretest and posttest level of leg muscle cramps.

\begin{tabular}{|l|c|c|c|c|c|}
\hline & Mean & SD & df & t value & Table value \\
\hline Pre test & 8.3 & 3.62 & 29 & 7.73 & 2.05 \\
\hline Post Test & 3.1 & - & - & - & - \\
\hline
\end{tabular}

of significance while comparing the Intradialytic stretching exercises and dextrose $25 \%$. Thus Intradialytic stretching exercises reduce the level of muscle cramps after 6 days of therapy.

These results are in agreement with Hallegraeff JM, et al. [23] who emphasized the effect of calf stretching exercises in the prevention of muscle cramps during hemodialysis. This study showed that there were no significant differences between the intradialytic stretching exercises and the routine treatment after 4 days of intervention. Only after 6 days of intervention, there was a significant difference at $\mathrm{p}<0.05$ level of significance.

The present study shows that there is effectiveness in the prevention and the reduction of muscle cramps during hemodialysis after longer days of therapy. This study concluded that continuous Intradialytic stretching exercises can prevent and reduces the level of muscle cramps during hemodialysis.

\section{Summary}

Muscle cramp is an unpleasant subjective feeling of suffering experienced by patient undergoing hemodialysis.

A study was conducted to assess the effectiveness of Intradialytic stretching exercises in leg muscle cramps among patients undergoing hemodialysis in the Ernakulum District. Prevalence of leg muscle cramps assessed by using cramp questionnaire and numerical pain scale score. 30 samples were selected for the study. Pre-test conducted by using standardized numerical pain scale, cramp questionnaire and demographic performance. Post-test conducted by using the same questionnaire and numerical pain scale. The leg muscle cramps reduced after Intradialytic stretching exercises.

\section{Conclusion}

Muscle cramps are found to be common in patient undergoing hemodialysis. Sometimes the leg muscle cramps affect the quality of life among patients undergoing hemodialysis and it also influences their daily activities. Every one undergoing hemodialysis are more susceptible to have leg muscle cramps. Almost $80 \%$ of patient undergoing hemodialysis will experience leg muscle cramps during or after hemodialysis. Various ways and means are available to prevent leg muscle cramp and related health problems of the patient undergoing hemodialysis. Intradialytic stretching exercise is one of the most important exercises to relieve leg muscle cramp. 


\section{Nursing implication}

The findings of the present study have an implication to nursing education among patients undergoing hemodialysis, nursing practice, nursing administration and nursing research.

\section{Nursing service}

- $\quad$ Nurse can provide health education regarding management of leg muscle cramps.

- Nurse can provide awareness programs about the new modalities of preventing leg muscle cramps such as exercise therapy, nutritional therapy, muscle pain therapy and vinegar therapy.

\section{Nursing administration}

- $\quad$ Periodically organize formal training program for nurses to know about the other alternative therapies.

- Nurse can arrange conference in service education, workshop which might be useful for the staffs.

- Encouraging the student and staff to disseminate the findings.

\section{Nursing education}

- Nursing curriculum should include content areas of various stretching exercises

- The educational authority should be advisable to incorporative Intradialytic stretching exercise to patients undergoing hemodialysis by involving nurses.

Nursing students should be equipped with the knowledge on the benefits of exercise.

\section{Nursing research}

- The study can be used as a reference material for further research regarding management of leg muscle cramps during dialysis.

- Research studies in this area will add to the body of knowledge of research, many nursing research studies can be derived from this topic.

\section{Suggestions}

- $\quad$ Patients undergoing dialysis should be aware about the leg muscle cramps and its complications.

- $\quad$ Educational programs should be conducted among patients undergoing hemodialysis regarding the effect of intradialytic stretching exercise to reduce leg muscle cramps.

\section{Limitations}

- $\quad$ The sample size was only 30 .

- The scope of generalization is limited.

- The period of data collection was limited.

- $\quad$ Purposive sampling technique was used for sample selection which limits the generalization of study.

- Sample collection was limited to one area of community.

\section{Recommendations}

- $\quad$ The present study can be replicated with more samples for generalization.

- The study can be done using other measures that help to reduce muscle cramps.
In the same study, the time period should be extended up to 8 sittings to 10 sittings to give intradialytic stretching exercises.

A planned teaching program can be conducted for patients undergoing hemodialysis on various methods to reduce leg muscle cramps.

\section{References}

1. Okwuonu CG, Chukwuonye II, Ogah SO, Abali C, Adejumo OA, et al. (2015) Awareness Level of Kidney Functions and Diseases among Adults in a Nigerian Population. Indian J Nephrol 25: 158-163.

2. Wasén E, Isoaho R, Mattila K, Vahlberg T, Kivelä SL, et al. (2004) Estimation of Glomerular Filtration Rate in the Elderly: A Comparison of Creatinine-Based Formulae With Serum Cystatin C. J Intern Med 256: 70-78.

3. Rothenbacher D, Klenk J, Denkinger M, Karakas M, Nikolaus T, et al. (2012) Prevalence and Determinants of Chronic Kidney Disease in Community-Dwelling Elderly by Various Estimating Equations. BMC Public Health 12: 343.

4. Zhang QL, Rothenbacher D (2008) Prevalence of Chronic Kidney Disease in Population-Based Studies: Systematic Review. BMC Public Health 8: 117.

5. Hemmelgarn BR, Zhang J, Manns BJ, Tonelli M, Larsen E, et al. (2006) Progression of Kidney Dysfunction in the Community-Dwelling Elderly. Kidney Int 69: 2155-2161.

6. Li ZY, Xu GB, Xia TA, Wang HY (2006) Prevalence of Chronic Kidney Disease in a Middle and Old-Aged Population of Beijing. Clin Chim Acta 366: 209-215.

7. Hansberry MR, Whittier WL, Krause MW (2005) The Elderly Patient With Chronic Kidney Disease. Adv Chronic Kidney Dis 12: 71-77.

8. Chadban SJ, Briganti EM, Kerr PG, Dunstan DW, Welborn TA, et al. (2003) Prevalence of Kidney Damage in Australian Adults: The AusDiab Kidney Study. J Am Soc Nephrol 14: S131-S138.

9. Malekmakan L, Haghpanah S, Pakfetrat M, Malekmakan A, Alimanesh M, et al. (2010) Dialysis Adequacy and Kidney Disease Outcomes Quality Initiative Goals Achievement in an Iranian Hemodialysis Population. Iran J Kidney Dis 4: 39-43.

10. Abbasi $Z$ (2013) The effect of Intradialytic stretching exercise on severity of symptoms of RLS and quality of sleep in hemodialysis patients.

11. World Kidney Day (2015) Chronic Kidney Disease.

12. Titze S, Schmid M, Köttgen A, Busch M, Floege J, et al. (2015) Disease Burden and Risk Profile in Referred Patients With Moderate Chronic Kidney Disease: Composition of the German Chronic Kidney Disease (GCKD) Cohort. Nephrol Dial Transplant 30: 441-451.

13. Chatrath $\mathrm{H}$ (2012) On association of prevalence and morbidity with muscle cramps in patients during hemodialysis session. Journal of AMJ 5: 127-128.

14. Kobrin SM, Berns JS (2007) Quinine--a Tonic Too Bitter for Hemodialysis-Associated Muscle Cramps? Semin Dial 20: 396-401.

15. Deschamps T (2016) Let's Programme Exercise During Haemodialysis (Intradialytic Exercise) into the Care Plan for Patients, Regardless of Age. Br J Sports Med 50: 1357-1358.

16. Parker K (2016) Intradialytic Exercise is Medicine for Hemodialysis Patients. Curr Sports Med Rep 15: 269-275. 
17. Maniam R, Subramanian P, Singh SK, Lim SK, Chinna K, et al. (2014) Preliminary Study of an Exercise Programme for Reducing Fatigue and Improving Sleep among Long-Term Haemodialysis Patients. Singapore Med J 55: 476-482.

18. Kouidi E, Karagiannis V, Grekas D, lakovides A, Kaprinis G, et al. (2010) Depression, Heart Rate Variability, and Exercise Training in Dialysis Patients. Eur J Cardiovasc Prev Rehabil 17: 160-167.

19. Dobsak P, Homolka P, Svojanovsky J, Reichertova A, Soucek M, et al. (2012) Intra-dialytic Electrostimulation of Leg Extensors May Improve Exercise Tolerance and Quality of Life in Hemodialyzed Patients. Artif Organs 36: 71-78.

20. Cheema BS, Smith BC, Singh MA (2005) A Rationale for Intradialytic Exercise Training as Standard Clinical Practice in ESRD. Am J Kidney Dis 45: 912-916.
21. Gowthami (2012) The effectiveness of Intradialytic stretching exercise on muscle Cramps among patients undergoing hemodialysis. Journal of TNNMU V 20: 40-43.

22. Mohamed M, Mohamed A, Abo Zead S (2007) Impact of Stretching Exercises Protocol on Reduction of Muscle Cramping during Haemdialysis, among Chronic Renal Failure Patients. Al-Azhar Assuit Medical Journal 5: 226-244.

23. Hallegraeff JM, de Greef MH, van der Schans CP (2013) An effective stretching regimen to prevent nocturnal leg cramps. Journal of Physiotherapy 59: 279-279. 